\title{
Clinical T4b Esophageal Cancer: Can We Make an "Unresectable" Tumour Resectable?
}

\author{
Lorenzo Ferri, MD, $\mathbf{P h D}^{1}$ \\ David S Mulder Chair in Surgery, Departments of Surgery and Oncology, Montreal General Hospital, McGill University, \\ Montreal, Quebec, Canada
}

Surgical resection of esophageal cancer, particularly in the context of a multimodality setting, represents the greatest chance for cure of this aggressive malignancy. However, tumours with either direct local invasion into what has long been perceived as "unresectable" structures (e.g., aorta, airway, or spine) have traditionally not been offered curative intent surgery. Although definitive chemoradiotherapy can be considered as a curative intent approach for some of these cT4b cancers (e.g., aorta or spine invasion), confirmed airway invasion, with a high risk of treatment related fistula, has in the past biased physicians towards an entirely palliative treatment approach, with dismal outcomes and survival measured in weeks. ${ }^{1,2}$ Challenging this paradigm, recent promising results from single-centre, retrospective studies have raised the possibility of curative intent esophagectomy for suspected cT4b with a reasonable long-term survival. ${ }^{3}$ The results of the COSMOS trial published in this edition of the Annals of Surgical Oncology attempt to clarify this controversial issue for these locally advanced, unresectable esophageal tumours through a prospective phase II study. ${ }^{4}$

Dr. Yokota and colleagues present the long-term data from the COSMOS trial, an important Japanese Clinical Oncology Group multi-institutional study investigating a stepwise curative intent approach of traditionally unresectable/incurable esophageal squamous cancer (ESCC). Induction chemotherapy was employed to identify

(C) Society of Surgical Oncology 2019

First Received: 1 August 2019;

Published Online: 22 August 2019

L. Ferri, MD, PhD

e-mail: lorenzo.ferri@mcgill.ca biologically favourable lesions for which curative intent conversion surgery could eventually be entertained. This trial included patients of good performance status with clinical T4b (aorta, airway, or spine invasion) or singlestation nonregional lymph node M1 (supraclavicular lymph nodes). After three cycles of docetaxel, cisplatin, and 5-flourouracil, patients were reassessed for surgical resection. If they were still deemed unresectable, then induction chemoradiotherapy (to $40 \mathrm{~Gy}$ ) was adopted to determine whether surgery could then be entertained. For those in whom the tumour was still unresectable, consolidation radiotherapy to $60 \mathrm{~Gy}$ was undertaken. With this logically ordered stepwise approach, the authors reported an impressive overall 3-year survival rate of more than $45 \%$ for this heterogeneous high-risk patient population. Understandably, the authors found an improved survival for the 19 of 48 (40\%) patients in whom they were able to convert to surgery with complete resection (R0) after induction therapy.

Dr. Yokota and his colleagues should be commended for attempting to clarify a controversial topic, the optimal treatment of locally unresectable esophageal cancer, through a prospective phase II trial. Although this manuscript is an important contribution to the literature, just like every good study, as many questions arise as are answered. The first is an issue of patient selection criteria for enrollment into the trial. The inclusion of both locally aggressive cT4b tumours and those with supraclavicular lymph nodes into the same study is a bit odd, because these are two very different diseases, but understandable given likely concerns of accrual. Positive supraclavicular lymph nodes imply the molecular propensity for metastatic dissemination and probable systemic disease; however, cT4b in the absence of clinically overt distant metastasis likely represents a different tumour biology with a molecular 
proclivity for local invasion. Nonetheless, in this trial, supraclavicular lymph node metastasis represented a minority of enrolled patients (9/48), the majority of which were cT4b with airway involvement (34/48). Thus, the conclusions of this study are likely more representative of the latter situation. This leads to a second issue that is not clarified by this present study; what does cT4b mean? The traditional definition of $\mathrm{T} 4 \mathrm{~b}$ implies invasion of an unresectable organ, but this can have a wide clinical spectrum. Indeed, for the present trial cT4b (airway) was defined as a tumour that "extended into the lumen or caused a deformity of the airway" (italics added). The first definition, with bronchoscopic biopsy confirmation of trans-airway invasion, is a very different scenario than the second and represents a much more complex therapeutic challenge due to the risk of fistula formation. A tumour with only extrinsic compression of the trachea, but no frank tracheal/ bronchial mucosal invasion, is at high risk of being overstaged. Although this important difference is not discussed in the manuscript, the surgical data presented seem to suggest that the majority, if not all, of the cT4b cases are simply suspected, rather than confirmed invasion of the trachea or bronchus. None of the procedures in this trial included en bloc resection of the airway or aorta, notwithstanding the chance for downstaging with induction therapy. It therefore is possible that a significant portion of the suspected cT4b lesions were in fact less aggressive T3 tumours. Although we and others have demonstrated anecdotally, in highly selected patients, that successful resection of these "unresectable" structures en bloc with the esophagus is feasible and can yield excellent long-term oncologic outcomes, the question of how to manage confirmed cT4b tumours with airway invasion remains unanswered by this present study.

As improved systemic therapies, both conventional cytotoxic and biologic/immunotherapy, become more readily available for esophageal squamous cell carcinoma, the opportunities to offer curative intent local therapy will continue to expand. Indeed, the use of a docetaxel-based triplet in this study seems to confer significant improvement comparted to the platin-based doublets used in the past. The approach for locally aggressive ESCC proposed by this present study, a period of induction docetaxel-based chemotherapy to select those that might benefit from local therapy (surgery \pm radiotherapy), is indeed very attractive given the dismal outcomes of those treated with palliative approaches alone. Moving forward, this study reinforces the notion that surgeons need to be engaged at all stages of esophageal cancer, and continually reengaged during the treatment trajectory, to ensure that patients who could potentially benefit from curative intent surgery are offered this approach.

\section{REFERENCES}

1. Ku GY, Goodman KA, Rusch VW, Ilson DH. Successful treatment of esophageal cancer with airway invasion with induction chemotherapy and concurrent chemoradiotherapy. J Thorac Oncol. 2009;4(3)432-4.

2. Nasir BS, Tahiri M, Kazakov J, et al. Palliation of concomitant tracheobronchial and esophageal diseases using a combined airway and esophageal approach. Ann Thor Surg. 2016;102(2):400-6.

3. Yamaguchi S, Morita M, Yamamoto M, et al. Long-term outcome of definitive chemoradiotherapy and induction chemoradiotherapy followed by surgery for T4 esophageal cancer with tracheobronchial invasion. Ann Surg Oncol. 2018;25(11):3280-7.

4. Yokota T, Kato K, Hamamoto Y, et al. Three-year overall survival update from a phase II study of chemoselection with DCF and subsequent conversion surgery for locally advanced unresectable esophageal cancer. Ann Surg Oncol. 2019 (in press).

5. Yokota T, Kato K, Hamamoto Y, et al. Phase II study of chemoselection with docetaxel plus cisplatin and 5-flourouracil induction chemotherapy and subsequent conversion surgery for locally advanced unresectable oesophageal cancer. Br J Cancer. 2016;115(11):1328-34

Publisher's Note Springer Nature remains neutral with regard to jurisdictional claims in published maps and institutional affiliations. 